\title{
Efeitos das tecnologias da informação na comunicação de pesquisadores da Embrapa
}

\author{
Maria da Paixão Neres de Souza \\ Doutora em Ciência da Informação. \\ Salvador - Bahia \\ Email: mariapaixaosouza@uol.com.br
}

\begin{abstract}
Resumo
Estudo realizado com 49 pesquisadores de nove unidades descentralizadas da Embrapa, contemplando dois centros por tema básico, três ecorregionais e quatro de pesquisa por produto (23\% das 39 unidades), no período de 1992 a 1997. A coleta de dados foi feita por meio de questionários eletrônicos, com retorno de 75 respondidos $(17 \%$ do total enviado). Os resultados mostraram melhoria nos contatos entre os pesquisadores, estabelecendo-se extensa rede de comunicação no país e no exterior. Observou-se a ocorrência, ainda em pequena escala, da avaliação e da publicação de trabalhos técnico-científicos em meio eletrônico. Conclui-se que o uso de tecnologias da informação afetou positivamente a comunicação científica do grupo estudado, com maior eficiência na produção, avaliação e difusão do conhecimento e, consequentemente, melhores resultados no campo da pesquisa e do desenvolvimento.
\end{abstract}

\section{Palavras-chave}

Comunicação científica; Tecnologia de informação; Fontes de informação; Produção de conhecimento; Avaliação de trabalhos; Difusão de conhecimento.

\section{The effects of the information technology in Embrapa's researchers communication systems}

\begin{abstract}
This is a study carried out with 49 researcers from nine decentralized units of Embrapa, comprising two centers by basic theme, three ecorregional and four of research by product (23\% of the decentralized units), from 1992 to 1997. The data collection was carried out by means of electronic questionnaires, with 75 replies (17\% out of the total sent). The results showed an improvement in the contacts among the researchers, setting up a wide network of communication in the country and overseas. Although in a small scale, evaluation and publication of technico-scientific works were carried out by electronic means. The conclusion is that the use of information technologies had a positive influence on the scientific communication of the group studied, having a greater efficiency in production, evaluation and dissemination of knowledge and, therefore, better results in the field of research and development.
\end{abstract}

\section{Keywords}

Scientific communication; Information technology; Information sources; Knowledge production; Referee; Knowledge diffusion.

\section{INTRODUÇÃO}

A análise das mudanças existentes quanto ao desenvolvimento do pensamento científico é fundamental para a compreensão das instituições científicas, considerando que o primeiro objetivo dessas instituições é a produção de novo conhecimento. Uma das primeiras questões sociológicas que devem ser levantadas é se a comunidade científica e a variação no padrão de comunicação entre cientistas afetam atualmente o desenvolvimento do conhecimento.

Embora essa questão tivesse sido levantada por Crane (1972, p.11) na década de 70, neste momento ela se torna extremamente oportuna para estudar as mudanças ocorridas no processo de produção, legitimação e difusão do conhecimento após a introdução do uso das tecnologias da informação na comunicação científica, visto que muitas das características do sistema de comunicação permanecem desconhecidas.

Reconhecida como a unidade básica de organização da ciência, a comunidade científica pode ser percebida pelos estudos de citação na literatura e pelos estudos de comunicação científica. No entanto, o processo de comunicação na ciência vem sofrendo sucessivas e significativas mudanças em decorrência da:

a) introdução das redes de computadores e das tecnologias de informação no ambiente de desenvolvimento do trabalho de pesquisa;

b) mudança de interesse dentro da própria ciência para o entendimento concreto dos sistemas e dos processos;

c) tendência atual voltada para a aplicação e uso, que é parte da reflexão do persistente interesse comercial e militar na ciência e na tecnologia.

A literatura tem mostrado que a comunicação foi bastante favorecida pelo uso das redes de computadores no ambiente de pesquisa. A comunicação interpessoal se

\footnotetext{
Aceito para publicação em artigo elaborado com base nos resultados da tese de doutorado "As tecnologias de informação no processo de produção, legitimação e difusão do conhecimento dos pesquisadores da Embrapa", defendida na Universidade de Brasília (UnB).
} 
tornou mais ágil, e foi ampliada a disponibilidade de informação pelo acesso às bibliotecas virtuais de todo o mundo. Houve incremento na troca de pré-publicações nas redes e estímulo para elaboração de publicações eletrônicas, contribuindo para que os resultados de pesquisa se tornem públicos com maior rapidez e para uma audiência mais ampla.

Além disso, a comunicação é imprescindível no modelo de produção, legitimação e difusão do conhecimento atual, no qual são considerados enfoques como transdisciplinaridade, heterogeneidade, controle de qualidade, imputabilidade social e reflexividade. Nessas configurações citadas por Gibbons et alii (1994), os elos entre os praticantes são mantidos, parcialmente, pelos canais formais (publicação, por exemplo) e, também parcialmente, pelos canais informais (troca de mensagens, reuniões técnicas, lista de discussão). Presume-se, portanto, que todas essas mudanças estão afetando o próprio modelo de produção, legitimação e difusão do conhecimento.

Diante disso, pretendeu-se verificar como o uso de tecnologias de informação afetou o sistema de comunicação de pesquisadores da Empresa Brasileira de Pesquisa Agropecuária (Embrapa), uma instituição vinculada ao Ministério da Agricultura que tem como missão viabilizar soluções para o desenvolvimento sustentável do agronegócio brasileiro por meio de geração, adaptação e transferência de conhecimentos e tecnologias, em benefício da sociedade.

\section{REVISÃO DE LITERATURA}

A comunicação científica foi definida por Bernal (1946, p.292-308) como um amplo processo de geração e transferência de informação científica. Nessa mesma linha de pensamento, Garvey (1979, p. IX), em trabalho cujo título é sugestivamente "Comunicação: a essência da ciência”, afirma que a comunicação científica inclui o espectro total de atividades associadas com a produção, a disseminação e o uso da informação, a partir do momento em que o cientista tem a idéia para sua pesquisa, até que a informação sobre os resultados desta pesquisa seja aceita como constituinte do conhecimento científico. Por comunicação científica o autor entende aquelas atividades de troca de informação que têm lugar principalmente entre os cientistas envolvidos em pesquisas de vanguarda, ou seja, no grupo de cientistas mais produtivos em determinada disciplina.
Várias crenças acerca da ciência implicam necessariamente sua ligação com interação social. A necessidade de acumular dados, desenvolver teorias e experiências em paralelo e modificar idéias tem por base a comunicação entre cientistas (Meadows, 1998, p.49).

Desde a fase de identificação do problema de pesquisa, entendido aqui como o processo de criar ou classificar novos conhecimentos, até a fase de assimilação do conhecimento gerado pelo beneficiário final, o pesquisador consulta várias fontes de informação.

Essas fontes podem ser classificadas como canais de comunicação informais e formais, como explicam Garvey \& Griffith (1967, p.1013). No domínio dos canais formais, Braga (1974, p.161) afirma que o documento e a comunicação formal representam apenas $20 \%$ no processo de geração do conhecimento. Referindo-se à comunicação utilizada pelos pesquisadores, acrescenta que $80 \%$ desta comunicação provêm de outros pesquisadores por meio de canais informais: conversas, conferências, seminários, cartas e outros tentáculos do colégio invisível.

Além dos tipos de canais utilizados para a divulgação, a velocidade com que esta divulgação ocorre é igualmente importante. Quanto mais rápido um projeto de pesquisa chama a atenção da comunidade científica e os seus resultados são divulgados, tanto melhor para o estado da ciência como um todo. A rapidez na divulgação faz com que descobertas sejam colocadas à disposição de outros pesquisadores no menor espaço de tempo possível, possibilitando que o conhecimento circule e sirva para novas pesquisas. A velocidade da divulgação move o progresso científico e propicia que seja assegurada a prioridade das descobertas (Stumpf, 1997, p.6).

Para atender à necessidade de maior velocidade quanto ao fluxo de produção de conhecimento, a rede de computadores e o uso das tecnologias de informação permitem a interação e colaboração entre indivíduos em localidades ainda que remotas, constituindo-se como a mais interessante alternativa para centralizar a geração e disseminação da informação, um paradigma de mudanças, portanto, para a construção e divulgação do conhecimento.

As tecnologias de informação e de comunicação mais usadas atualmente incluem telefone, fax, correio eletrônico, videoconferência, grupos de discussão, salas interativas (chats). Por conta dessas tecnologias, a troca 
de informação (texto, dados, imagens) ocorre de forma virtual e em tempo real. Em conseqüência, permite o desenvolvimento simultâneo da troca de idéias e discussão dos resultados de pesquisa em vários locais, conduzindo a conclusões em colaboração com outros cientistas. Assim, o ambiente eletrônico oferece um novo campo de oportunidades para o processo de comunicação científica, que, para funcionar a contento, necessita do suporte que as telecomunicações e as tecnologias de computação têm a oferecer.

\section{PESQUISA DE USO DAS TECNOLOGIAS}

Considerando que a Embrapa intensificou o processo de adoção de tecnologias de informação a partir de 1991, a pesquisa teve como objetivo examinar o efeito dessas tecnologias na comunicação científica de seus pesquisadores. $\mathrm{O}$ estudo teve uma abrangência de seis anos, compreendendo o período de 1992 a 1997.

Para a realização do estudo, foram selecionadas aleatoriamente nove unidades de pesquisa das 39 que a Embrapa possui, representando $23 \%$ do total das unidades, ficando assim distribuídas: dois centros temáticos - a) Embrapa Agroindústria Tropical e b) Embrapa Agroindústria de Alimentos; quatro Centros de produto - a) Embrapa Hortaliças, b) Embrapa Milho e Sorgo, c) Embrapa Soja e d) Embrapa Trigo; três Centros ecorregionais - a) Embrapa Cerrados, b) Embrapa SemiÁrido e c) Embrapa Amazônia Oriental.

O universo da pesquisa constou de 49 pesquisadores (empregados da Embrapa classificados no Plano de Cargos e Salários dentro dessa categoria), selecionados aleatoriamente, de cada uma das nove unidades da amostra, perfazendo um total de 441 pesquisadores, ou seja, $21 \%$ do contingente da empresa.

Para a coleta dos dados, foi enviado um questionário por meio eletrônico para cada um dos 441 pesquisadores, facultada a devolução pela forma mais conveniente para o entrevistado: meio eletrônico, correio ou malote da empresa (Anexo).

Todas as variáveis usadas foram tabuladas, e os dados foram analisados empregando-se o Statistical Package for the Social Sciences (SPSS), versão 8.0 para Windows. Essas variáveis foram objeto da análise de freqüência simples, freqüência cruzada e de análises descritivas.

\section{RESULTADOS DA PESQUISA}

Dos 441 questionários enviados para os pesquisadores, foram recebidos 75 questionários, representando uma taxa de retorno de $17 \%$. A maioria dos questionários foi devolvida por meio eletrônico - $54,67 \%$ pela Web, $28 \%$ por correio eletrônico -, e apenas $17,33 \%$ foram devolvidos pelo malote da Embrapa. Portanto, esse baixo índice de retorno pode ser um indicativo de que só aqueles que aderiram ao uso efetivo da Internet para condução dos seus trabalhos de pesquisa tenham devolvido o questionário respondido. Além desse, outros problemas inclusive de ordem técnica podem também ter interferido no aspecto de devolução dos questionários, conforme atestaram alguns dos entrevistados.

As respostas às questões propostas ao grupo pesquisado indicam a maneira como o sistema de comunicação desse grupo foi afetada pelo uso das tecnologias de informação. Elas são apresentadas nos próximos itens.

\section{Rede de comunicação entre pesquisadores}

Primeiramente, pretendeu-se, com a pesquisa, identificar a existência de uma rede de comunicação ou um colégio invisível (o grupo de colegas com os quais o pesquisador troca idéias na condução do seu trabalho de pesquisa) entre o grupo de pesquisadores, solicitando indicação dos colegas com quem o pesquisador entrevistado trocava idéias sobre atividades profissionais.

Os dados mapeados foram:

a) pesquisadores da mesma unidade da Embrapa;

b) pesquisadores de outra unidade da Embrapa;

c) pesquisadores de outras instituições;

d) se o colega indicado era do país ou do exterior;

e) área de especialização desse colega .

Na tabela 1, a seguir, são mostrados os dados de afiliação dos colegas com quem o pesquisador entrevistado troca idéias.

Os dados indicam que $62,2 \%$ dos entrevistados trocam idéias de pesquisa com colegas na mesma unidade. Embora essa comunicação ocorra na mesma unidade, verificou-se que $54,2 \%$ dos entrevistados trocam idéias de pesquisa com colegas das mais diversas áreas do conhecimento agrícola, como apresentado na tabela 2 , a seguir. 
Tal fato pode ser um indício de heterogeneidade no modo de produzir conhecimento. No enfoque da heterogeneidade, os problemas de pesquisa estão sendo resolvidos por especialistas em disciplinas diversas. Essa tendência retrata o atual modelo de produção, legitimação e difusão do conhecimento, como afirmam Gibbons et alii (1994).

A despeito de alguns membros do grupo da amostra terem feito seu curso de pósgraduação no exterior, 96,6\% dos colegas com quem os entrevistados trocam idéias trabalham no Brasil, o que poderia ser explicado pela afirmação de Alves (1980, p.9) de que a tecnologia do setor agrícola é tão específica quanto a local. Raramente, pode ser transferida de um país para outro, ou de uma região para outra, por causa das peculiaridades da agricultura, a exemplo do solo, clima, tipo de cultura característica de cada região, daí a comunicação endógena.

Entretanto, quando se observa que foram indicados 232 colegas com quem o grupo troca idéias de pesquisa, verifica-se que os dois sujeitos mais citados o foram apenas quatro vezes, o que representa 5,8\% dos casos do total de indicações, sendo a maioria indicada apenas uma vez. Os colegas mais indicados são identificados como os gatekeepers pesquisadores que formam o elo entre as fontes de informação e os colegas.

Todos esses dados relacionados com a comunicação na ciência vêm demonstrar o quanto é importante o colégio invisível, principalmente no contexto da transdisciplinaridade, no qual a comunicação entre os pares é fundamental na elaboração da agenda de pesquisa e no enriquecimento do debate científico realizado via rede eletrônica.

\section{Fontes de informação para a produção de conhecimento}

Conforme os dados da tabela 3, a fonte de informação mais utilizada para a produção de conhecimento são as bibliotecas, com um percentual de $80 \%$; seguida

TABELA 1

Afiliação do colega que atua na rede de comunicação

$\begin{array}{lccc}\text { Categoria } & \text { Código } & \text { Freqüência } & \text { \% Respostas } \\ \text { Mesma unidade da Embrapa } & 1 & 150 & 62,2 \\ \text { Outra unidade da Embrapa } & 2 & 17 & 7,1 \\ \text { Outra instituição } & 3 & 74 & 30,7 \\ \text { Total de respostas } & & 241 & 100\end{array}$

Nota: 70 casos válidos, cinco sem resposta.

\section{TABELA 2 \\ Heterogeneidade no sistema de comunicação científica da Embrapa}

Categoria

Mesma área e unidade

Mesma área e outra unidade

Área diferente e mesma unidade

Área diferente e outra unidade

Mesma área e outra instituição no Brasil

Mesma área e outra instituição no exterior

Area diferente e outra instituição no Brasil

Total de respostas

Nota: 69 casos válidos; seis sem resposta.
Código \% Freqüência \% Respostas

TABELA 3

Fontes de informação utilizadas pelos pesquisadores para produção de conhecimento

$\begin{array}{llllll}\text { Categoria } & \text { Mais usado } & \text { Uso regular } & \text { Pouco usado } & \text { Não respondeu } & \text { Total } \\ & \mathrm{f}(\%) & \mathrm{f}(\%) & \mathrm{f}(\%) & \mathrm{f}(\%) & \\ \text { Bibliotecas } & 60(80 \%) & 14(18,7 \%) & 0 & 1(1,3 \%) & 75 \\ \text { Colegas } & 11(14,7 \%) & 37(49,3 \%) & 23(30,7 \%) & 4(5,3 \%) & 75 \\ \text { Fontes impressas } & 43(57,3 \%) & 29(38,7 \%) & 0 & 3(4 \%) & 75 \\ \text { Fontes eletrônicas } & 17(22,7 \%) & 30(40 \%) & 23(30,7 \%) & 5(6,7 \%) & 75 \\ \text { Eventos } & 25(33,3 \%) & 35(46,7 \%) & 13(17,3 \%) & 2(2,7 \%) & 75 \\ \text { Ambiente de trabalho } & 12(16 \%) & 39(52 \%) & 17(22,7 \%) & 7(9,3 \%) & 75 \\ \text { Outras fontes } & 6(8 \%) & 5(6,7 \%) & 4(5,3 \%) & 60(80 \%) & 75\end{array}$

de fontes impressas, com 57,3\%; eventos (congressos, seminários), com 33,3\%. Em quarto lugar, aparece fontes eletrônicas, com um índice de $22,7 \%$; ambiente de trabalho, com $16 \%$, colegas, com $14,7 \%$ das indicações, sendo a penúltima fonte de produção de conhecimento. A literatura tem demonstrado que cientistas citam sempre os colegas como fonte de produção de conhecimento. De acordo com esses resultados, os pesquisadores estão lendo mais que conversando com os colegas, um comportamento mais próximo dos que se utilizam da informação tecnológica para a produção de conhecimento. 
Em estudo com questões semelhantes a essas, realizado com um grupo de agrônomos, Rolinson, AlShanbari e Meadows (1996, p.49-50) encontraram um percentual de $39 \%$ para o item comunicação com os colegas e $55 \%$ destinado à leitura. Contudo, quando os autores solicitaram a indicação de outras fontes de informação, os cientistas britânicos, objeto do estudo, apontaram que discussão com colegas é para eles a maior fonte de informação, corroborando outros estudos sobre o assunto.

\section{Freqüência de uso dos canais de informação}

O telefone é o canal de informação mais utilizado pelo grupo (32\% das respostas), e correio aéreo/ terrestre e serviço de distribuição de documentos (malote) obtiveram igualmente 6,7\% de respostas.

Tais resultados concordam com o levantamento realizado por Rolinson, Al-Shanbari \& Meadows (1996, p.53). Os autores identificaram que, além desses canais, o uso crescente do correio eletrônico e abstracts/índices eletrônicos são indicadores de mudanças claramente evidentes.

Essas mudanças também podem ser verificadas nesse grupo após a adoção das tecnologias de informação. Fontes tradicionais cederam lugar a outras que permitem o rápido acesso à informação técnico-científica, evidenciadas na questão sobre mensagens veiculadas no meio eletrônico.

\section{Tipo de mensagem veiculada}

Para o grupo de pesquisadores analisados, mensagens de cunho geral é o tipo mais usado no meio eletrônico, com $62,7 \%$ das respostas, depois informação científica-tecnológica, com $46,7 \%$ das respostas, e informações bibliográficas, com 22,7\% (tabela 4).

Resultados semelhantes aparecem no trabalho de Figueira Netto (1994, p.74) sobre a comunicação científica via de redes de computadores, entre pesquisadores que usavam a rede há mais de dois anos. Nesse trabalho, os serviços mais utilizados foram mensagens gerais, com um percentual de uso de $47 \%$, informação científica, com 36\%, informação tecnológica, 30\%, e informação sobre cursos e eventos, com $25 \%$ de respostas.
Nessa questão, observa-se ainda baixo percentual de pesquisadores $(33,3 \%)$ que indicaram usar com regularidade a rede para fazer revisão de trabalhos. Esta é uma prática que tem sido adotada na comunidade científica e que proporciona agilidade na disponibilidade do conhecimento, quando elimina os entraves do correio comum e, posteriormente, da impressão em papel.

\section{Freqüência de uso dos meios eletrônicos}

Com que freqüência os meios eletrônicos são utilizados para a produção, legitimação e difusão do conhecimento? Das respostas, 94,7\% indicam que o correio eletrônico é utilizado diariamente, seguido de transferência de arquivo, com $16 \%$. Outros meios têm uso diário inexpressivo. Contudo, é importante ressaltar que, embora com baixos índices de uso, todos os meios apresentados na questão são utilizados pelo grupo estudado. Presumese que, em estudos posteriores, esse quadro terá uma outra situação, ou seja, a adoção de tecnologias da informação e comunicação seja mais expressiva.

De acordo com os resultados de Figueira Netto (1994, p.74), os serviços mais utilizados são nesta ordem: correio eletrônico, com um percentual de 64\%; listas de discussão, com 37\%; transferência de arquivo e netnews, com 37\%; fórum/teleconferência, com um percentual de $24 \%$. Vale transcrever também o depoimento que o autor obteve de um pesquisador sobre os fóruns de discussão na rede: "Fóruns eletrônicos têm sido vitais para mim. Através do correio eletrônico, tenho podido contactar muito mais pesquisadores do que seria possível, mesmo indo a três congressos por ano em diversas partes do mundo". 
Freqüência do tipo de uso dos meios eletrônicos pelos pesquisadores

Conforme as categorias dos tipos de uso identificadas na tabela 4 , a freqüência diária aponta mensagens pessoais como a mais utilizada, com $58,7 \%$ das respostas. Depois, aparece troca de idéias de pesquisa, com $30,7 \%$ das respostas, e ainda merece destaque fornecer ajuda técnica $(14,7 \%)$. Quanto aos tipos de uso com freqüência semanal, a busca de literatura obteve $40 \%$, o maior índice de respostas, e, para itens com realização mensal, destaca-se avaliação de trabalhos técnico-científicos, com $40 \%$ das respostas, conforme a tabela 5 .

\section{Uso de meio eletrônico em atividades de difusão de tecnologia}

Os resultados mostraram que, para as atividades de difusão de tecnologia, publicações, geração de tecnologia, inovação tecnológica, comunicação por meio do rádio e da televisão, entre outras, os pesquisadores utilizam muito pouco o meio eletrônico. Ainda são raras as publicações na rede de computadores, e, ainda assim, esta parece ser a única iniciativa do grupo.

Pelo visto, a situação permanece a mesma tal qual foi constatada por Quirino, Cruz \& Souza (1993, p.69). Em estudo sobre a produção do conhecimento na pesquisa agropecuária, esses autores analisaram projetos de pesquisa dos quais listaram seis tipos de ações diferentes para difusão de tecnologia. Como resultado, o estudo indicou que os mecanismos de transferência menos utilizados foram os referentes à mídia eletrônica.

\section{Fatores de uso da comunicação eletrônica que contribuíram para a melhoria do trabalho de pesquisa}

O uso das tecnologias de informação contribuiu para melhorar o trabalho de pesquisa. $\mathrm{O}$ grupo citou diversos fatores que evidenciam esta afirmação. A principal mudança observada após a introdução do uso das tecnologias de informação referiram-se em sua maioria a: a) rapidez na comunicação, com 49,3\% das respostas;

b) atividades da comunicação científica (conhecimento de novos grupos e linhas de pesquisa; acesso ao diálogo na comunidade científica; troca de experiência com parcerias e novas pesquisas; contatos rápidos com experts em todo o mundo; publicação de trabalhos científicos; facilidade para redigir projetos, relatórios e comunicações; organização de palestras; aumento do universo de contatos ) obtiveram 37,3\% das respostas;

c) eficiência na comunicação, com 26,9\% das respostas;

d) melhoria de comunicação, com 20,9\% das respostas.

Em estudo semelhante, sobre a rede de informação na universidade, Budd e Connaway (1997, p.847) encontraram $60 \%$ de respostas afirmando que a sua comunidade disciplinar foi submetida a mudanças recentes como resultado da rede de informação. Alguns acadêmicos ofereceram indicações de que suas comunidades foram expandidas geograficamente após o uso da comunicação eletrônica, e 162 dos entrevistados relataram que a sua comunidade se tornou mais interdisciplinar.

\section{CONSIDERAÇÕES FINAIS}

Os resultados mostraram que o uso de tecnologias da informação afetou o sistema de comunicação desse grupo de pesquisadores. As mudanças observadas são percebidas à medida que, graças ao uso de tecnologias de informação, confirma-se a atuação em uma grande rede de comunicação formada por colegas da Embrapa e, em menor número, 
por colegas de outras instituições que atuam principalmente no país. Essa configuração pode ser denominada o colégio invisível dessa comunidade.

Neste colégio invisível eletrônico utilizado como prática na comunicação científica pelos pesquisadores entrevistados, são veiculadas informações técnicocientíficas, bibliográficas, cursos e eventos. O grupo utiliza o correio eletrônico diariamente, participa de listas de discussão e faz revisão/avaliação de trabalho.

As transformações ficaram patentes quando a maioria informou que o maior benefício do uso das tecnologias de informação foi a rapidez na comunicação e a melhoria na comunicação científica, proporcionando o conhecimento de novos grupos e linhas de pesquisa, acesso ao diálogo na comunidade científica, troca de experiência com parcerias e novas pesquisas.

Observou-se que, na área de difusão do conhecimento publicações, inovações tecnológicas -, são poucas as atividades que se utilizam do meio eletrônico. Portanto, pode-se concluir que as tecnologias de informação são mais utilizadas na produção de conhecimento do que na avaliação e na difusão de conhecimento. Ainda assim, há evidências de que o uso das tecnologias de informação transformou a comunicação científica do grupo estudado, fazendo com que a produção, a avaliação e a difusão de conhecimento sejam conduzidas de maneira mais eficiente, contribuindo para o alcance dos resultados no campo da pesquisa e desenvolvimento.

Artigo aceito para publicação em 24-5-2002

\section{REFERENCIAS}

ALVES, E. R. de. A Embrapa e a pesquisa agropecuária no Brasil. Brasília : Embrapa, Departamento de Informação e Documentação, 1980. 22 p. (Embrapa. Documentos, 2).

BERNAL, J. D. The social function of science. London : George Routledge, 1946. 482 p. cap. 11, p. 292-308.

BRAGA, G. M. Informação, ciência, política científica: o pensamento de Derek de Solla Price. Ciência da Informação, Rio de Janeiro, v. 3, n. 2, 155-177, 1974.

BUDD, J. M.; CONNAWAY, L. S. University faculty and network information: results of a survey. Journal of the American Society for Information Science, v. 48, n. 9, p. 843-852, 1997.

CRANE, D. Invisible colleges: diffusion of knowledge in scientific communities. Chicago : University of Chicago, 1972. 127p.

FIGUEIRA NETTO, S. C. A comunicação científica através de redes de computadores: a experiência de pesquisadores brasileiros. 1994. $161 \mathrm{f}$. Dissertação (Mestrado em Ciência da Informação) - IBICT, UFRJ, Rio de Janeiro, 1994.

GARVEY, W. D. Communication: the essence of science. Oxford : Pergamon, 1979. 332 p.

; GRIFFITH, B. C. Scientific communication as a social system. Science, v. 157, p. 1011-1016, 1967.

GIBBONS, M. et al. The new production of knowledge: dynamics of science and research in contemporary societies. London : Sage Publications, 1994. 179 p.

MEADOWS, A. J. Communicating research. San Diego, California : Academic Press, 1998. 266 p. (Library and Information Science).

QUIRINO, T. R.; CRUZ, E. R da; SOUZA, G. S. A produção do conhecimento na pesquisa agropecuária. Revista de Administração, São Paulo, v. 28, n. 4, p .62-72, 1993.

ROLINSON, J.; AL-SHANBARI, H.; MEADOWS, A. J. Information usage by biological researchers. Journal of Information Science, v. 22, n. 1, p. 47-53, 1996.

SOUZA, M. P. N. de. As tecnologias da informação no processo de produção, legitimação e difusão do conhecimento dos pesquisadores da Embrapa. 1999. 209 f. Tese (Doutorado em Ciência da Informação) - Universidade de Brasília, Brasília, 1999.

STUMPF, I. R. C. A comunicação da ciência na UFRGS: relato de pesquisa. Porto Alegre : UFRGS, 1997. 21 p. 


\section{ANEXO}

\section{QUESTIONÁRIO}

Prezado(a) Pesquisador(a):

Sou lotada na Embrapa Mandioca e Fruticultura e estou realizando curso de Doutorado em Ciência da Informação na Universidade de Brasília, conduzindo trabalho de pesquisa para a elaboração da tese, cujo tema é a influência das tecnologias de informação na produção, legitimação e difusão do conhecimento dos pesquisadores da Embrapa. Para a obtenção dos dados para esta pesquisa, estou conduzindo um levantamento mediante um questionário, entre os pesquisadores de sua unidade. O questionário requer cerca de 10 a 20 minutos do seu tempo para ser respondido, e eu prometo manter e respeitar a confiabilidade das informações prestadas por V.Sa. Portanto, peço a sua colaboração para responder a todas as questões formuladas e devolver o questionário por meio eletrônico ou para a Área de Informação da Sede da Embrapa, a atenção da Chefe da AI-Sede, Simara Gonçalves. Agradeço-lhe e ponho-me à disposição para qualquer esclarecimento.

\section{Maria da Paixão Neres de Souza paixao@sede.embrapa.br}

\section{A. IDENTIFICACÃO}

1) Nome:

2) Unidade:

3) Endereço eletrônico:

4) Faixa etária:

$$
\begin{aligned}
& 4.1(\text { ) 20-24 anos } \\
& 4.2(\text { ) 25-29 anos } \\
& 4.3(\text { ) 30-34 anos } \\
& 4.4(\text { ) 35-39 anos }
\end{aligned}
$$

$4.5($ ) $40-44$ anos
$4.6($ ) $45-49$ anos
$4.7($ ) $50-54$ anos
$4.8($ ) 55-59 anos
$4.9($ ) mais de 60

5) Nível de escolaridade, local e ano de conclusão de cada curso:

\begin{tabular}{|l|l|l|}
\hline Nivel de escolaridade & local & Ano de conclusão \\
5.1 ( ) graduação & & \\
5.2 ( ) mestrado & & \\
5.3 ( ) doutorado & & \\
5.4 ( ) pós-doutorado & & \\
\hline
\end{tabular}

\section{B. ATIVIDADES PROFISSIONAIS}

1) Há quanto tempo exerce a função de pesquisador?

2) Qual é a sua especialização profissional?

3) Quais são as culturas com as quais você trabalha?

\section{INFORMAC̣ÃO E COMUNICAC̣ÃO}

1) Qual é o colega com quem você troca idéias sobre as atividades profissionais? (Em caso de haver mais de um, cite-os por ordem de freqüência maior para a menor)

Nome do colega

Nome da instituição

2) Quais as fontes de informação que você consulta para produzir conhecimentos? Use códigos: M (muito usado); R (usado com regularidade); P (pouco usado):

2.1 ( ) bibliotecas

2.2 ( ) colegas

2.3 ( ) fontes impressas

2.4 ( ) fontes eletrônicas

2.5 ( ) eventos (congressos, seminários)

2.6 ( ) ambiente de trabalho

2.7 ( ) outros (especifique)

3) Indique a freqüência de uso no seu trabalho de pesquisa dos seguintes canais de informação. Use códigos: a (nunca); b (menos que 1 vez/mês); c (1-4 vezes/mês); $\mathrm{d}$ (1-5 vezes/semana); e (diariamente).

3.1 ( ) correio aéreo/terrestre

3.2 ( ) telefone

3.3 ( ) telex

3.4 ( ) fax

3.5 ( ) serviço de distribuição de documentos

3.6 ( ) reuniões

4) Que tipo de mensagem eletrônica é veiculada? Use códigos: M (muito); R (usado com regularidade); P (pouco usado).

4.1 ( ) informação científica-tecnológica

4.2 ( ) informações bibliográficas

4.3 ( ) informações sobre cursos/eventos

4.4 ( ) mensagens de cunho geral

4.5 ( ) revisão/avaliação de trabalhos científicos

4.6 ( ) outros (especifique) 
5) Com que freqüência você usa os seguintes meios eletrônicos? Use códigos: D (diária); S (semanal); M (mensal); N (nunca)

5.1 ( ) correio eletrônico

5.2 ( ) listas de discussão

5.3 ( ) transferência de arquivos

5.4 ( ) chats

5.5 ( ) CD-ROM

5.6 ( ) teleconferência

5.7 ( ) consultas a bibliotecas virtuais

5.8 ( ) análise de dados de pesquisa

5.9 ( ) análise de reações químicas

5.10( ) simulação de atividades de pesquisa

5.11( ) outros (especifique e quantifique)

6) Indique a freqüência de cada tipo de uso. Use códigos: d (diária); s (semanal); m (mensal); n (nunca).

6.1 ( ) mensagens pessoais

6.2 ( ) reuniões

6.3 ( ) administração de projetos

6.4 ( ) troca de idéias de pesquisa

6.5 ( ) receber ajuda técnica

6.6 ( ) fornecer ajuda técnica

6.7 ( ) busca de literatura

6.8 ( ) intercâmbio de documentos

6.9 ( ) conferencias eletrônicas

6.10 ( ) avaliação de trabalhos técnico-científicos

6.11 ( ) outros (especifique)
7) Qual o meio utilizado para divulgação de cada subprojeto? Responda quantificando.

Código do subprojeto:

7.1 ( ) programa de rádio

7.2 ( ) programa de televisão

7.3 ( ) jornal

7.4 ( ) feiras

7.5 ( ) base de dados

7.6 ( ) revista de divulgação

7.7 ( ) outros (especifique)

8) Cite por ordem de importância pelo menos três fatores de melhoramento para o seu trabalho de pesquisa resultado do uso da comunicação eletrônica:

a)

b)

c) 